\title{
Erratum to: Clinical impact of albuminuria and glomerular filtration rate on renal and cardiovascular events, and all-cause mortality in Japanese patients with type 2 diabetes
}

\author{
Takashi Wada - Masakazu Haneda Kengo Furuichi $\cdot$ Tetsuya Babazono $\cdot$ Hiroki Yokoyama \\ Kunitoshi Iseki · Shin-ichi Araki · Toshiharu Ninomiya · Shigeko Hara • Yoshiki Suzuki • \\ Masayuki Iwano $\cdot$ Eiji Kusano $\cdot$ Tatsumi Moriya $\cdot$ Hiroaki Satoh $\cdot$ Hiroyuki Nakamura \\ Miho Shimizu - Tadashi Toyama $\cdot$ Akinori Hara $\cdot$ Hirofumi Makino $\cdot$ The Research Group of \\ Diabetic Nephropathy, Ministry of Health, Labour, and Welfare of Japan
}

Published online: 11 February 2014

(C) Japanese Society of Nephrology 2014

\section{Erratum to: Clin Exp Nephrol \\ DOI 10.1007/s10157-013-0879-4}

During the editorial process a difference occurred in the layout of Table 4 between the PDF and HTML versions, whereas no difference or error actually exists in the data. The correct layout for the table is shown here to avoid any possible misunderstanding for readers. The correction of this layout involves no change whatsoever in the data shown in the table.

The online version of the original article can be found under doi:10.1007/s10157-013-0879-4.

T. Wada $\cdot$ K. Furuichi $\cdot$ M. Shimizu $\cdot$ T. Toyama $\cdot$ A. Hara Division of Nephrology, Kanazawa University Hospital,

Kanazawa, Japan

T. Wada $(\bowtie)$

Division of Nephrology, Department of Laboratory Medicine, Institute of Medical, Pharmaceutical and Health Sciences,

Faculty of Medicine, Kanazawa University, Kanazawa,

13-1 Takara-machi, Kanazawa 920-8641, Japan

e-mail: twada@m-kanazawa.jp

\section{Haneda}

Department of Medicine, Asahikawa Medical University,

Asahikawa, Japan

K. Furuichi $\cdot$ M. Shimizu $\cdot$ T. Toyama $\cdot$ A. Hara

Department of Disease Control and Homeostasis, Institute of

Medical, Pharmaceutical and Health Sciences, Kanazawa

University, Kanazawa, Japan

T. Babazono

Division of Nephrology and Hypertension, Diabetes Center,

Tokyo Women's Medical University School of Medicine,

Tokyo, Japan
H. Yokoyama

Jiyugaoka Medical Clinic, Internal Medicine, Obihiro, Japan

K. Iseki

Dialysis Unit, University Hospital of the Ryukyus, Nishihara, Okinawa, Japan

S. Araki

Department of Medicine, Shiga University of Medical Science, Otsu, Shiga, Japan

T. Ninomiya

Department of Medicine and Clinical Science, Graduate School of Medical Sciences, Kyushu University, Fukuoka, Japan

S. Hara

Center of Health Management, Toranomon Hospital, Tokyo, Japan

Y. Suzuki

Health Administration Center, Niigata University, Niigata, Japan 
Table 4 Hazard ratios based on levels of UACR and eGFR for each outcome

The estimates are adjusted for age, gender, HbA1c, systolic BP

\begin{tabular}{|c|c|c|c|}
\hline \multirow[b]{2}{*}{ UACR } & \multicolumn{3}{|c|}{$\operatorname{eGFR}\left(\mathrm{ml} / \mathrm{min} / 1.73 \mathrm{~m}^{2}\right)$} \\
\hline & $>60$ & 30-59 & $<30$ \\
\hline \multicolumn{4}{|c|}{ Renal events (RRT or halving reduced eGFR) } \\
\hline Normoalbuminuria & \multicolumn{2}{|l|}{1.00 (Reference) } & \multirow{3}{*}{$49.82(29.9-83.0)$} \\
\hline Microalbuminuria & \multicolumn{2}{|l|}{$3.26(2.34-4.55)$} & \\
\hline Macroalbuminuria & $13.6(9.3-20.0)$ & $33.0(22.7-48.2)$ & \\
\hline \multicolumn{4}{|l|}{ Cardiovascular events } \\
\hline Normoalbuminuria & \multicolumn{2}{|l|}{1.00 (Reference) } & \multirow{3}{*}{$1.54(1.00-2.39)$} \\
\hline Microalbuminuria & \multicolumn{2}{|l|}{$1.40(1.16-1.69)$} & \\
\hline Macroalbuminuria & $1.90(1.36-2.65)$ & $2.09(1.54-2.84)$ & \\
\hline \multicolumn{4}{|l|}{ All-cause mortality } \\
\hline Normoalbuminuria & \multicolumn{2}{|l|}{1.00 (Reference) } & \multirow{3}{*}{$7.08(4.16-12.05)$} \\
\hline Microalbuminuria & \multicolumn{2}{|l|}{$1.30(0.93-1.81)$} & \\
\hline Macroalbuminuria & $2.34(1.35-4.04)$ & $4.59(2.90-7.25)$ & \\
\hline
\end{tabular}

M. Iwano

Division of Nephrology, Department of General Medicine,

University of Fukui, Fukui, Japan

E. Kusano

Division of Nephrology, Department of Internal Medicine, Jichi Medical University, Tochigi, Japan

\section{T. Moriya}

Health Care Center, Kitasato University, Sagamihara, Japan

H. Satoh

Department of Nephrology, Hypertension, Diabetology, and

Metabolism, Fukushima Medical University, Fukushima, Japan
H. Nakamura

Department of Environmental and Preventive Medicine, Institute of Medical, Pharmaceutical and Health Sciences, Kanazawa University, Kanazawa, Japan

\section{H. Makino}

Department of Medicine and Clinical Science, Okayama University Graduate School of Medicine, Dentistry and

Pharmaceutical Sciences, Okayama, Japan 